\title{
Nano-imaging and Its Applications to Biomedicine
}

\author{
Elisabetta Canetta ${ }^{1, *}$ and Ashok K. Adya ${ }^{2}$ \\ ${ }^{1}$ Cardiff University, School of Biomedical Sciences, Museum Avenue, PO Box 911, \\ Cardiff, CF10 3US, Wales, UK \\ ${ }^{2}$ University of Abertay Dundee, Division of Biotechnology and Forensic Sciences, \\ Bell Street, Dundee, DD1 1HG, Scotland, UK \\ CanettaE@cardiff.ac.uk, ashok.adya@abertay.ac.uk
}

\begin{abstract}
Nanotechnology tools, such as Atomic Force Microscopy (AFM), are now becoming widely used in life sciences and biomedicine. AFM is a versatile technique that allows studying at the nanoscale the morphological, dynamic, and mechanical properties of biological samples, such as living cells, biomolecules, and tissues in their native state under physiological conditions. In this article, an overview of the principles of AFM will be first presented and this will be followed by discussion of some of our own recent work on the applications of AFM imaging to biomedicine.
\end{abstract}

Keywords: Atomic Force Microscopy, AFM, AFM imaging, elastic images, elastic modulus, biomedicine, cells, yeasts, biomolecules.

\section{Introduction}

Atomic force microscopy (AFM) was invented by Binning, Quate and Gerber in 1986 [1] and since then its use as a surface characterization tool has increased dramatically. In particular, in the last ten years AFM has become one of the most powerful nanotools in biology [2,3]. This success is due to the ability of the AFM to allow studying the structure, function, properties, and interaction of biological samples in their native state under physiological buffer conditions. Moreover, the excellent signal-to-noise ratio of the AFM allows obtaining images with sub-nanometer $(<1$ $\mathrm{nm}$ ) resolution which permits to investigate the ultrastructure of a broad range of samples ranging from cells (micrometer sized) to DNA and proteins (a few nanometers in size) [4]. Another advantage of AFM lies in minimal sample preparation requirements that preserve the real features of the specimens without damaging them or introducing any artifacts, thereby making AFM a very attractive and versatile nano-imaging tool in biological sciences [5].

The ability of AFM to visualize nanoscale topography and morphology of tissues, cells, and biomolecules permits a direct observation of the fine structural details of, e.g. cell surfaces, surface proteins, and DNA coiling. Processing of AFM images permits one to measure at the nanoscale, the surface roughness, texture, dimensions, and volumes of biological systems. This capability along with the ability of AFM to

${ }^{*}$ Corresponding author. 
detect pico-Newton force interactions between cells (cell-cell interaction), proteins (protein-protein interaction), a cell and a functionalized substrate (cell-substrate interaction), or a receptor and a ligand (lock-and-key interaction) has opened up completely new areas for investigating the mechanical and microrheological properties of biological samples, such as their adhesion, elasticity (property of a material which causes it to be restored to its original shape after applying a mechanical stress), viscosity (resistance of a fluid to deform under shear stress), and viscoelasticity (when materials change with time they respond to stress as if they are a combination of elastic solids and viscous fluids) [3].

In this paper, some of the relevant results by AFM obtained by the authors in the biomedical field will be presented. The computational processing of AFM images to obtain multiparameter analysis of cells and biomolecule functions will also be discussed.

\section{Principles of AFM}

The operating principle of an AFM is based upon scanning a fine tipped probe (called cantilever) just above a sample surface (Fig. 1) and monitoring the interaction force between the probe and the surface [3]. The cantilever obeys the Hooke's law, $F=k \cdot x$, where $F$ is the force on the cantilever, $k$ its spring constant, and $x$ its deflection. This relationship permits one to obtain the tip-sample interaction force, provided that the spring constant of the cantilever is known and its deflection measured. To measure the cantilever deflection a laser beam is reflected off the back side of a reflective (gold- or aluminium-coated) cantilever towards a position sensitive detector which can measure both normal bending and torsion of the cantilever, corresponding to normal and lateral forces.

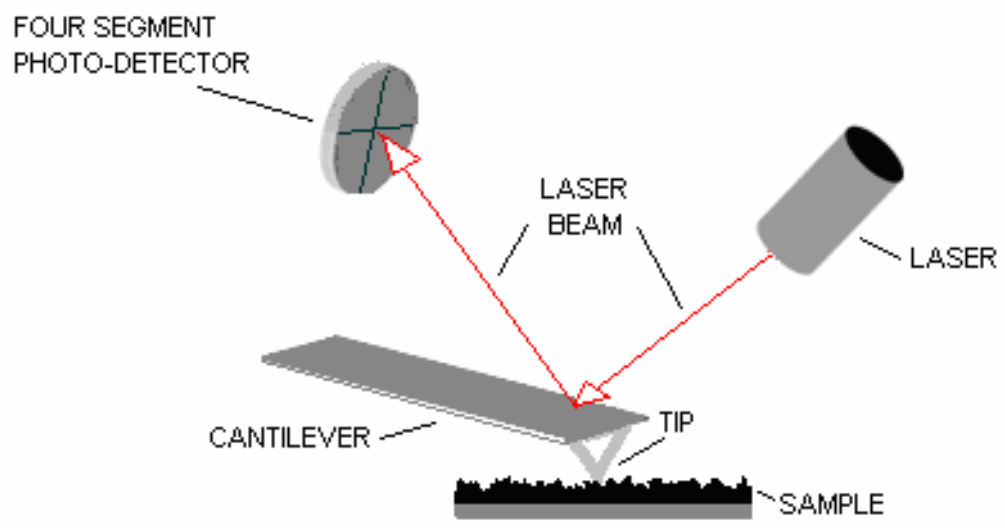

Fig. 1. Sketch of the operational principle of AFM 


\subsection{Application Modes of AFM}

Imaging. During scanning of the sample surface, the deflection of the cantilever is measured and used as an input signal for the feedback circuitry to maintain constant the tip-sample distance. By recording the displacements of the tip along the $z$-axis it becomes possible to build the topographic (height) image of the sample surface. A variety of operational modes, such as contact mode, and intermittent-contact tapping mode are available to image a sample surface [3].

Force Spectroscopy. The main outcome of a force spectroscopy experiment is the force-distance (F-d) curve, which shows the force experienced by the cantilever both when the AFM probe is brought in contact with the sample surface (trace/approach) and separated from it (retrace/retract) (Fig. 2). Analysis of the F-d curves with suitable theoretical models can give information on the elastic and adhesive properties of the sample. In particular, the maximum adhesive force of the sample to the tip $\left(F_{\max }\right)$, the energy of adhesion $\left(W_{a d h}\right)$, and the Young's modulus $(E)$ can be measured (Fig. 2).

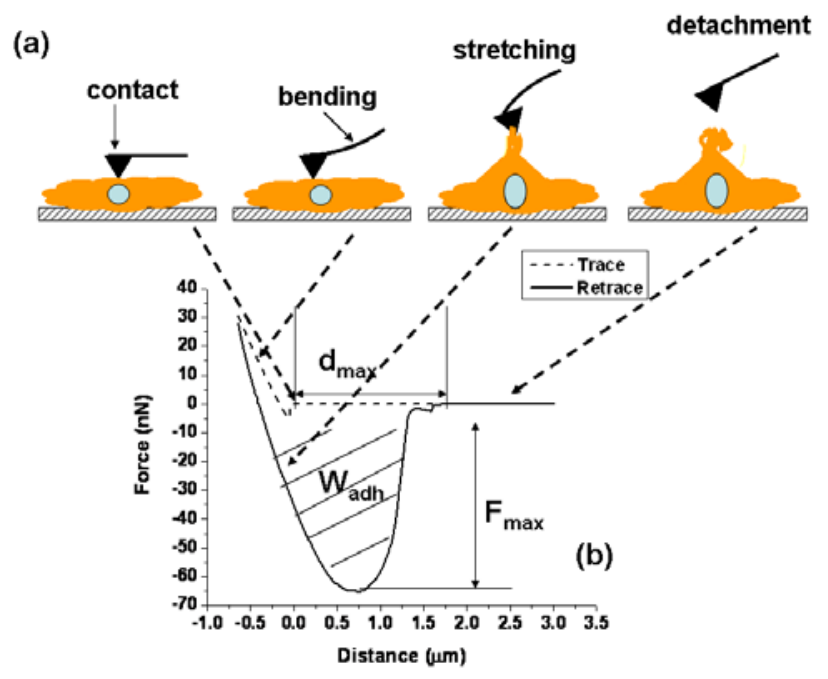

Fig. 2. (a) Sketch of the stages of a force spectroscopy experiment. Contact between the AFM probe and the sample surface and cantilever bending with AFM probe indentation occurs during the trace (approach) cycle of the AFM experiment. On the retrace (retract) cycle, the sample surface is stretched until the point of detachment. An example of a typical experimental $F-d$ curve for a trace and retrace cycle is also presented; the stages are identified by the arrows. The meanings of $F_{\max }, d_{\max }$, and $W_{a d h}$ are also identified.

Force Mapping. It combines force measurements and imaging [6,7]. A force mapping data set contains an array of force curves and a sample image as well. It can be used to obtain a 2D map of the nanomechanical properties (e.g. elasticity) of the 
sample surface (Fig. 3). It is worth noting that force spectroscopy and force mapping can be done only in contact mode.

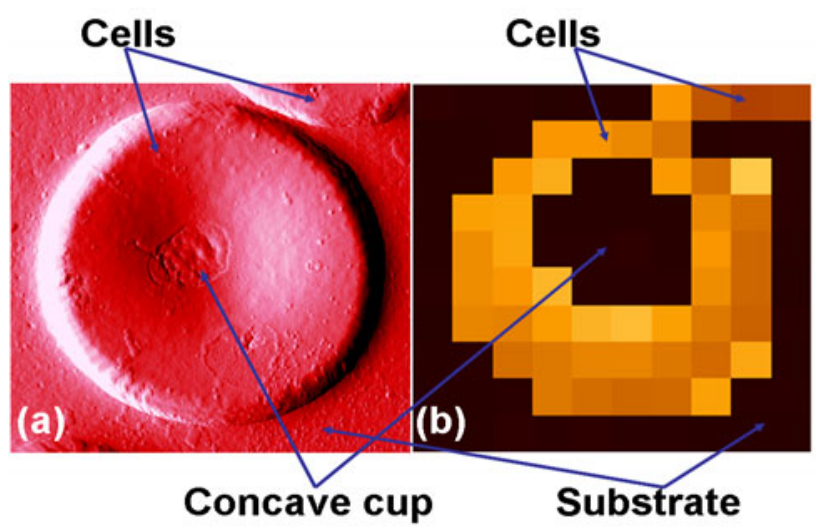

Fig. 3. AFM force mapping on a red blood cell (RBC). (a) AFM image of RBC (typical biconcave shape) on glass. (b) AFM elastic image of the RBC shown in (a). The difference in elasticity of the substrate and the cell surface allow obtaining an elastic map of the sample.

\section{Processing of AFM Images}

AFM experiments generate a wealth of data very quickly. In order to extract useful information from the data, each AFM image has to be carefully processed to remove sample tilt, scanner and probe geometry artifacts, and obtain correct image contrast and scaling. In addition, statistical evaluation of cross-sectional and surface texture analyses can be performed on AFM images to gain knowledge on the dimensions, surface roughness and texture, topographical profile and nanomechanical properties of the samples.

Image Enhancement. It is the first step in image processing and it mainly consists in: (i) leveling the raw image to take care of any tilt and bow that may be introduced to the image by the AFM scanner. This is achieved via background subtraction such as line-by-line or plane leveling; (ii) sharpening the raw image with high-pass filters to enhance the finest details of the sample surface topography; and (iii) smoothing the raw image to remove any background noise from the image. Here we wish to add a word of caution for the novice to be extremely careful because image enhancement, if not done properly, can introduce additional artifacts in the image.

Section Analysis. AFM images can be analysed by section analysis to determine the dimensions (e.g. length, width), and peak-to-valley heights of the sample (Fig. 4). In AFM images, horizontal dimensions of objects are usually greatly overestimated due to the well-known effect of tip convolution. Therefore, statistical methods should be used to correct the tip-convolution effects in AFM images in order to measure the real lateral dimension of the sample [8]. 

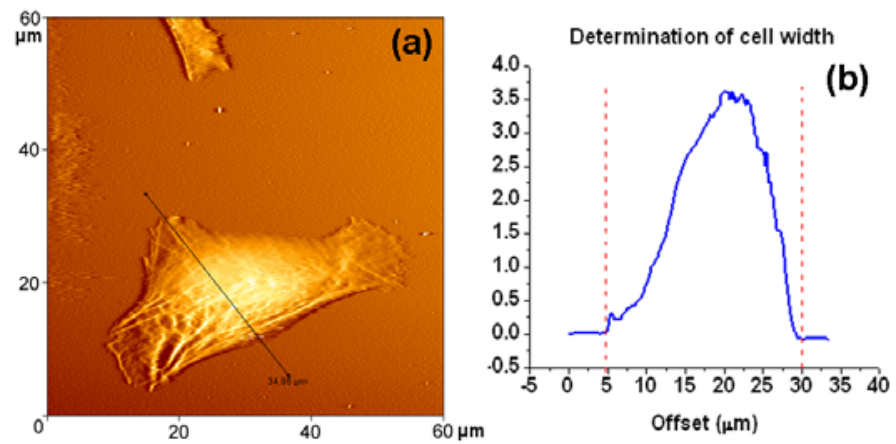

Fig. 4. (a) Section analysis on the AFM image of a living human urothelial cell (HUC) under physiological conditions, and (b) measure of the peak-to-valley height of the cell from crosssection analysis

Roughness Analysis. The roughness of the surface of a sample can be analysed by measuring the root mean square roughness, $R_{r m s}$, on a height image, defined as the standard deviation from the mean data plane of the $h$ (height) values of the AFM images within a selected region on the cell surface,

$$
R_{r m s}=\sqrt{\frac{\sum_{i=1}^{N}\left(h_{i}-\bar{h}\right)^{2}}{N}} .
$$

In Eq. (1), $h_{i}$ is the current height value, $\bar{h}$, the height of the mean data plane, and $N$, the number of points within the selected region of a given area. Roughness analysis is usually carried out on raw AFM images, i.e., images that are neither flattened nor elaborated with any filter (e.g., low pass or high pass filter).

\section{Applications of AFM to Imaging of Biological Systems}

Over the past 10 years, we have extensively used AFM in both imaging and force spectroscopy modes to investigate the ultrastructural and nanomechanical properties of different biological systems [9-11]. Some of our recent results on cells and biomolecules will be presented in the next sections.

\subsection{Discrimination between Candida albicans and Candida dubliniensis Cells under Normal and Pathological Conditions}

Candida albicans is a diploid fungus and a causal agent of opportunistic oral and genital infections in humans. Candida dubliniensis is commonly isolated from oral cavities and it is very closely related to $C$. albicans because both these yeasts are 
dimorphic. Dimorphism is defined as the switching between two cell-types. Both $C$. albicans and $C$. dubliniensis when they infect host tissues, switch from the usual unicellular yeast-like form into an invasive, multicellular filamentous form. Due to their dimorphic nature it is sometimes difficult for clinicians to distinguish between the two Candida and therefore to prescribe the more appropriate antifungal drug.

We used AFM imaging to investigate the ultrastructures of $C$. albicans and $C$. dubliniensis (isolated and sub-cultured from patients) after growing them in normal $\left(30^{\circ} \mathrm{C}\right)$ and pathogenic $\left(39^{\circ} \mathrm{C}\right)$ conditions. As shown in Figs. $5 \mathrm{a}$ and $5 \mathrm{~b}$, at $30^{\circ} \mathrm{C} \mathrm{C}$. albicans and $C$. dubliniensis cells remained non-pathogenic and they grouped together in colonies. However, increase in temperature led to the appearance of pathogenic cells for both strains of Candida (Figs. 5c and 5d) with the typical formation of hyphae.
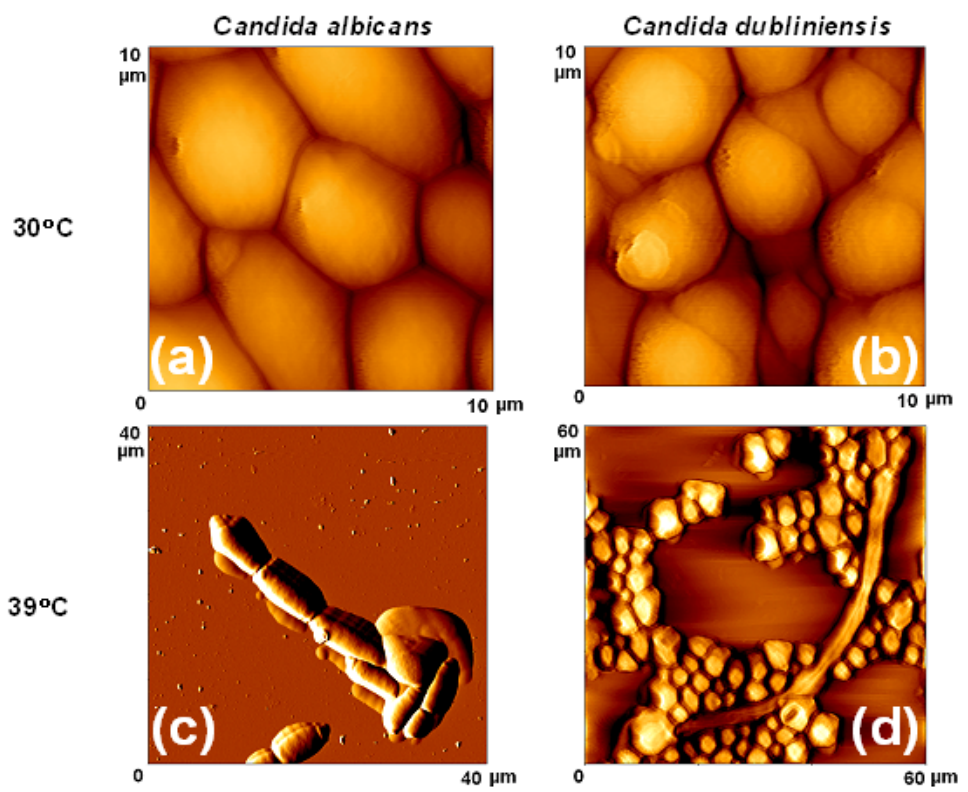

Fig. 5. AFM images of living C. albicans (Left) and C. dubliniensis (Right) yeast cells under physiological conditions, grown in non-pathogenic conditions at $30^{\circ} \mathrm{C}$ (upper row) and in pathogenic conditions at $39^{\circ} \mathrm{C}$ (bottom row). Height ranges in $\mu \mathrm{m}$ : (a) $0-4.3$, (b) $0-3.2$, (c) 0 $5.0,(\mathrm{~d}) 0-4.3$

Section analysis on the AFM images of the two types of Candida showed that for both $C$. albicans and $C$. dubliniensis, non-pathogenic cells were smaller than pathogenic cells. This difference could probably be due to the beginning of the process of extrusion of hyphae from the pathogenic yeast cells that resulted in an increase in their sizes. The main difference between $C$. albicans and $C$. dubliniensis revealed by the section analysis concerned their sizes. $C$. dubliniensis cells were 
found to be smaller than $C$. albicans. Moreover, the morphological structures of the hyphae of these two strains of Candida were quite different. In pathogenic C. albicans cells the hyphae were short $(21.2 \pm 3.1) \mu \mathrm{m}$, and they were divided in segments with well defined secta formed between the segments. On the contrary, in pathogenic $C$. dubliniensis the hyphae were long $(52.1 \pm 8.4) \mu \mathrm{m}$, and the secta dividing the segments were not as evident as in C. albicans.

\subsection{Determination of Elasticity of Human Embryonic Stem Cells}

Human embryonic stem cells (hESCs) are pluripotent, and they represent a promising source of cells for regenerative medicine. In a real clinical setting, the patient will receive mature, differentiated cells after they have been subjected to an appropriate differentiation protocol. However, most protocols result in a mixture of cells enriched for the desired population, but containing subpopulations of other cell types.

Separation and purification methods scalable to quantities of cells suitable for human cell replacement therapy are a major problem for stem cell therapy. Extensive biochemical and biomechanical characterisation of hESCs would be of great utility since such features might provide options for cell separation without requiring the use of antibodies as specific lineage markers. Cell elasticity is an important biomechanical parameter, which is primarily determined by the presence, number and distribution of specific organelles (e.g. nucleus, mitochondria) and the character and organisation of cytoskeletal elements (e.g. microfilaments, microtubules and intermediate filaments). It is well known that cell elasticity varies with cell function. Particular elasticities can also be associated with specific phenotypes. We employed AFM to determine the Young's (or elastic) modulus of hESCs. Single cell surfaces were mapped by performing AFM mapping experiments which allowed local variations due to cell structure to be identified.

AFM images showed the presence of two different phenotypes in the hESCs cell lines: (i) small and round (Fig. 6a) and (ii) large and spread (Fig. 6b). This difference could suggest that one phenotype corresponded to differentiated hESCs while the other to undifferentiated hESCs, but which one corresponded to what phenotype? A possible answer to this question was given by the Young's modulus values obtained from the AFM force mapping experiments. It was observed that the elasticity of hESCs varied at different points on the cell surface, reflecting their heterogeneous nature. In addition, variations in elasticity between cells that were larger than the variations on the individual cells were also observed. Such large variation in elasticity within hESCs of a particular cell line when compared to the variation across a single cell suggested significant structural differences between these cells. In particular, it was found that cells corresponding to phenotype (i) had higher Young's moduli, (8.16 $\pm 6.18) \mathrm{kPa}$, and thus they were stiff. However, cells corresponding to phenotype (ii) had lower Young's moduli, $(0.0485 \pm 0.0239) \mathrm{kPa}$, indicating that these cells were softer. These results seemed to suggest that stiffer cells might be differentiated while the softer ones might be undifferentiated, indicating a potential strategy for separation of differentiated and undifferentiated embryonic stem cells. 

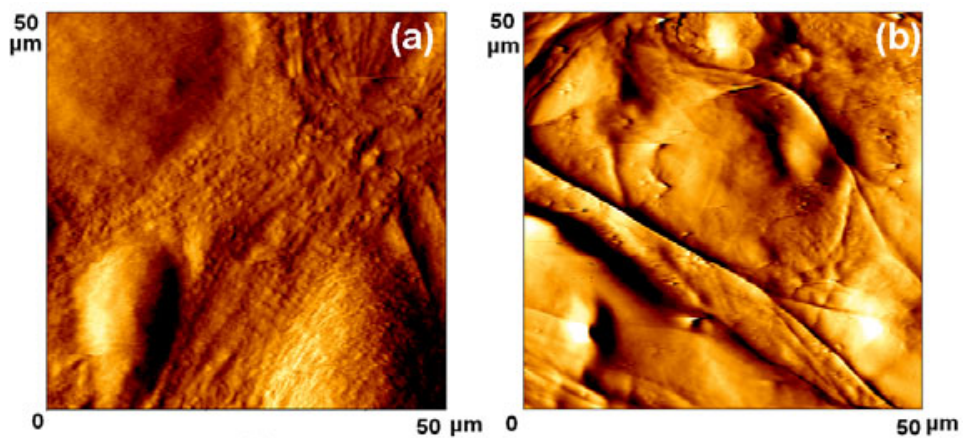

Fig. 6. AFM images of living hESCs in physiological conditions of probably (a) undifferentiated $(0-4.9 \mu \mathrm{m}$ height range) and (b) differentiated $(0-2.4 \mu \mathrm{m}$ height range $)$ hESC cells

\subsection{The Effects of Ionising Radiation on the Nanostructure of Pericardium Tissues}

The pericardium is a protective membrane that surrounds the heart and it is composed of collagen and elastin fibres. Its functions are related to the mechanical and structural properties of the heart. The effects of ionising radiation, with respect to cardiac doses received in breast radiotherapy, on the nanostructure of fibrous pericardium tissue were investigated by AFM imaging. 40Gy is the dose limit placed on $100 \%$ of the heart volume in a clinical radiotherapy department, and it is considered to be the threshold at which pericarditis occurs. Therefore, a range of doses lower than 40Gy, as well as one of $80 \mathrm{~Gy}$ was chosen to examine any structural changes of the pericardium under extreme conditions.

The control sample clearly showed (Fig. 7) that collagen fibrils bunched together and run parallel to one another. Fibrils had a distinct banding pattern, consisting of peaks and grooves, known as D-staggered configuration. Although, the AFM images do not seem to highlight a huge difference in the nanostructure of fibrils after exposure to ionising radiation, the section analysis carried out on the images showed that mean fibril width increases with radiation dose, with 80Gy having the largest mean fibril thickness by far, i.e., $(111.2 \pm 4.3) \mathrm{nm}$, compared with $(88.1 \pm 2.8) \mathrm{nm}$ for the control tissue. Quite surprisingly, no threshold at 40Gy was found. The higher the radiation dose, the larger the amount of fibres that became affected and the more swollen the affected fibres became, ultimately increasing the mean collagen fibre width for that sample. In addition, the D-period of the fibrils in both the un-irradiated and irradiated tissues was found to be remarkably consistent over the range of doses, indicating that the banding period, and hence axial molecular arrangement, was unaffected by irradiation. 

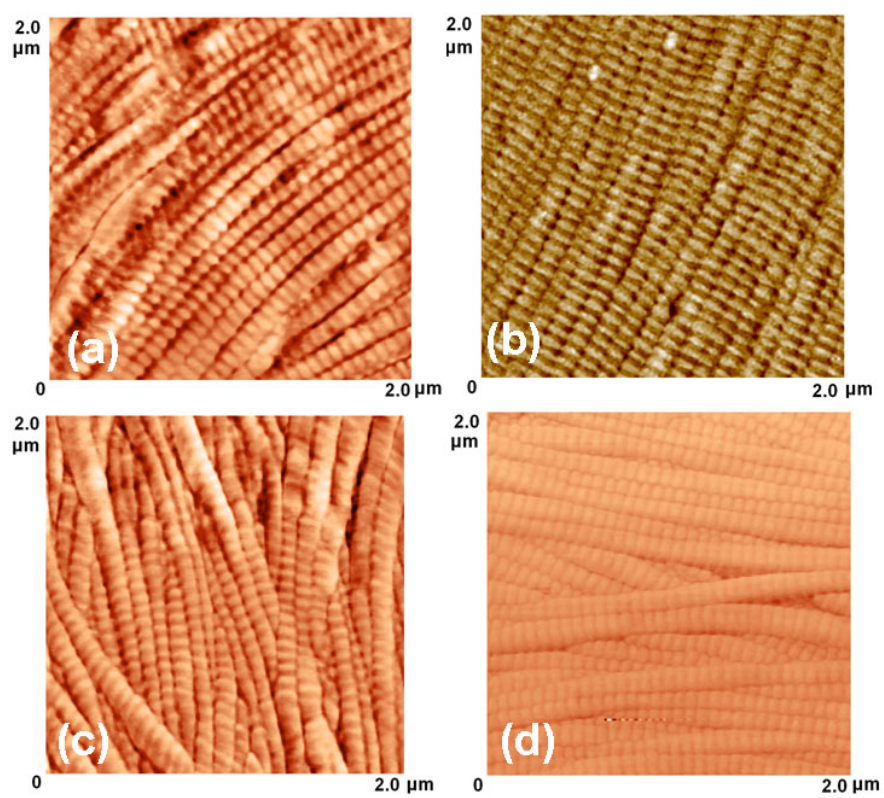

Fig. 7. AFM images of fibrous pericardial tissues exposed to (a) 0 Gy radiation $(0-400 \mathrm{~nm})$; (b) 5 Gy radiation $(0-450 \mathrm{~nm})$; (c) 40 Gy radiation $(0-525 \mathrm{~nm})$; (d) 80 Gy radiation $(0-650 \mathrm{~nm})$ with - height ranges of AFM images given within brackets

These results showed that ionising radiation caused the collagen fibrils to increase in diameter, with the number of fibrils affected and the extent of swelling increasing with the radiation dose. Moreover, because one of the main mechanisms by which radiation interacts with proteins is through the formation of cross-links, it is very likely that the increased fibril width observed in the AFM images of pericardium tissue was due to the formation of cross-links within the collagen fibrils. The formation of cross-linking in fibrils could possibly account for the fact that the Dperiod value was retained for most of the irradiated samples, indicating that when cross-links were formed in fibrils, the molecules followed the regular arrangement of the natural collagen.

\section{Conclusions}

AFM has emerged as a very powerful technique in biomedicine for investigating the ultrastructural and nanomechanical properties of cells and biomolecules. This success is mainly due to the ability of AFM to provide high-resolution images without causing any damage during sample preparation and scanning. In addition, the multivariate array of image processing tools allow making direct measurements of the dimensions, profiles, surface roughness, volumes, and nanomechanics (e.g. elasticity and adhesion) of the samples. Recent developments in the technology to produce higher scan rates (e.g. collection of images in less than 1s) have pushed forward the design of control software [12] with new and very user-friendly user interfaces. These new 
developments of AFM will certainly boost even further its use in clinical settings as a tool for disease screening and diagnosis.

\section{References}

1. Binning, G., Quate, C.F., Gerber, C.: Atomic Force Microscope. Phys. Rev. Lett. 56, 930933 (1986)

2. Ikai, A.: A Review on: Atomic Force Microscopy applied to Nano-Mechanics of the Cell. Adv. Biochem. Eng. Biotechnol. 119, 47-61 (2010)

3. Canetta, E., Adya, A.K.: Atomic Force Microscopy: Applications to Nanobiotechnology. J. Indian Chem. Soc. 82, 1147-1172 (2005)

4. Francis, L.W., Lewis, P.D., Wright, C.J., Conlan, R.S.: Atomic Force Microscopy comes of age. Biol. Cell, 133-143 (2010)

5. Fletcher, D.A., Mullins, R.D.: Cell Mechanics and Cytoskeleton. Nature 463, 485-492 (2010)

6. Haga, H., Sasaki, S., Kawabata, K., Ito, E., Ushiki, T., Sambongi, T.: Elasticity Mapping of Living Fibroblasts by AFM and Immunofluorescence Observation of the Cytoskeleton. Ultramicroscopy 82, 253-258 (2000)

7. Jung, Y.J., Park, Y.S., Yoon, K.J., Kong, Y.Y., Park, J.W., Nam, H.G.: Molecule-Level Imaging of Pax6 mRNA Distribution in Mouse Embryonic Neocortex by Molecular Interaction Force Microscopy. Nucl. Acids Res. 37, e10 (2009)

8. Schiffmann, K., Fryda, M., Goerigk, G., Lauer, R., Hinze, P.: Correction of STM Tip Convolution Effects in Particle Size and Distance Determination of Metal-C:H Films. Fresenius J. Anal. Chem. 358, 341-344 (1997)

9. Canetta, E., Walker, G.M., Adya, A.: Nanoscopic Morphological Changes in Yeast Cell Surfaces Caused by Oxidative Stress: An Atomic Force Microscopic Study. J. Microbiol. Biotechn. 19, 547-555 (2009)

10. Krysmann, M.J., Funari, S., Canetta, E., Hamley, I.W.: The Effect of PEG Crystallization on the Morphology of PEG-peptide Block Copolymers Containing Amyloid $\beta$ Peptide Fragments. Macromol. Chem. Physic. 209, 883-889 (2008)

11. Canetta, E., Duperray, A., Leyrat, A., Verdier, C.: Measuring Cell Viscoelastic Properties Using a Force-Spectrometer: Influence of Protein Cytoplasm Interactions. Biorheology 42, 321-333 (2005)

12. Carberry, D.M., Picco, L., Dunton, P.G., Miles, M.J.: Mappifn Real-Time Images of HighSpeed AFM Using Multitouch Control. Nanotechnology 20, 434018-434023 (2009) 